II Bulletin d'analyse phénoménologique XV 6, 2019

ISSN 1782-2041 https://popups.uliege.be/1782-2041/

DOI: $10.25518 / 1782-2041.1098$

\title{
Textes posthumes IV : Sur la théorie de l'objet : Natorp versus Husserl
}

\author{
Par ROBERT BRISART \\ Université Saint-Louis à Bruxelles, Université du Luxembourg
}

Résumé Partant de la critique natorpienne de Husserl, l'auteur explique en quel sens les Recherches logiques ont amorcé un démantèlement du «mythe de l'objet ou du donné », que Husserl cependant, en raison de son correspondantisme, n'a pas su mener à son terme avant la Krisis, où, se rapprochant étonnamment des idées de Natorp, il finit par reconnaître que « l'objectivité à laquelle prétendent les prestations de la science continuent de porter la marque du "subjectif-relatif", puisque toutes les productions scientifiques se rattachent ultimement à l'activité concrète de l'homme dans son monde ».

Mots-clefs constructivisme, Husserl, idéalisme, Natorp, néokantisme, réalisme, vérité.

Sous le titre de psychologie et les allures d'une analyse de la vie du sujet, nombre de travaux philosophiques importants de la seconde moitié du $\mathrm{XIX}^{\mathrm{e}}$ et du début du $\mathrm{XX}^{\mathrm{e}}$ ont eu pour souci d'éclairer ce que veut dire être un objet et se présentent donc également comme des essais d'ontologie. Tel est très certainement le cas de la psychologie empirique de Brentano, de celle aussi à laquelle Husserl donna le nom de phénoménologie et il ne semble pas en aller autrement de la psychologie selon la méthode critique que Natorp avait déjà conçue en 1888 et qu'il remit sur le métier pour une nouvelle mouture publiée en $1912^{1}$.

${ }^{1}$ P. Natorp, Psychologie générale selon la méthode critique, trad. franç. d’É. Dufour et J. Servois, Paris, <Vrin, > 2007. 
Quelle est donc la thèse concernant les objets que recèle la Psychologie de Natorp? Sans doute peut-elle être résumée de façon fort simple : s'il faut entendre par objet un donné quelconque qui préexisterait à la conscience et avec lequel celle-ci aurait à s'accorder pour devenir à proprement parler connaissance, alors l'objet est un mythe et un mythe non moins tenace que le préjugé qui le fonde. À l'inverse, il s'agit de soutenir que l'objet «n'est absolument pas en dehors de la conscience $»^{1}$. Sans doute n'en faut-il pas plus pour immédiatement verser la thèse de Natorp au compte de l'idéalisme. Cependant, cet idéalisme ne consiste pas à nier l'existence du réel pour le dissoudre dans l'esprit, ce que Natorp dénonce lui-même comme une malversation subjectiviste $^{2}$. Il consiste plutôt à soutenir que l'existence du réel ne fait pas encore l'existence des objets, et que si c'est une chose de reconnaître la réalité, tout autre chose est de présupposer qu'elle soit déjà faite d'entités déterminées et identifiables comme telles. A contrario, ce qu'on doit entendre par réalisme ne consiste pas simplement à défendre le principe de réalité, car, à ce compte-là, il serait difficile de ne pas être réaliste. En fait, le réalisme philosophique constitue une thèse beaucoup plus forte qui avec le réel engage aussi l'existence d'un ensemble d'objets déjà constitués par eux-mêmes et par rapport auxquels la correspondance de la représentation déciderait de sa validité. Si, en regard de cette thèse, l'idéalisme a de la pertinence pour Natorp, c'est d'abord qu'il apporte quelques arguments sérieux pour plaider en faveur d'un antiréalisme radical.

Quelles bonnes raisons avons-nous donc d'être antiréalistes en philosophie ? Natorp nous semble l'indiquer notamment en soulevant le problème que pose la thèse du remplissement qui, dans les Recherches logiques, constitue la version husserlienne du concept de vérité, laquelle est, à bien y regarder, une thèse éminemment correspondantiste et, partant, une thèse profondément réaliste. Sur ce point, Natorp oppose d'abord à Husserl que, si tant est qu'on puisse envisager quelque chose comme le remplissement d'une représentation de la conscience par la présentation d'un objet et qui vaudrait comme critère de la vérité de la représentation, ce remplissement en tout cas «n'a pas lieu en une fois », car, ajoute Natorp, « jamais, il n'y a et ne peut y avoir de remplissement absolu $»^{3}$. Mais alors, que peut bien vouloir dire que le remplissement de la représentation par l'objet est processuel, puisqu'il n'a jamais lieu en une fois, et qu'il est de surcroît relatif, puisqu'il n'est jamais absolu ? Aborder ces

\footnotetext{
${ }^{1}$ Ibid., XI $\S 13$, p. 319.

2 Ibid., VIII § 9, p. 235.

${ }^{3}$ Ibid., XI § 10, p. 320.
} 
questions, même dans les limites d'un débat avec Husserl, nécessite sans doute de creuser plus avant la teneur de l'antiréalisme natorpien.

\section{Le mythe des objets}

Profondément ancrée en nous existe ce qu'on appelle aujourd'hui une psychologie du sens commun ou plus simplement encore une psychologie naïve qui nous dote d'une certaine idée de nous-mêmes en première personne comme aussi d'une certaine idée du monde environnant. Même s'il n'est pas toujours aisé d'en expliciter les principes fondamentaux tant ils sont flous, on ne peut que reconnaître à cette psychologie une extraordinaire efficacité, puisque, grâce à elle, nous semblons prémunis d'un nombre assez considérable d'assurances qui manifestement régulent de façon très fiable nos relations avec les autres et avec les choses. Aux autres, cette psychologie permet d'attribuer des états mentaux plus ou moins semblables à ceux qui nous sont propres, de lire quasiment en eux pour, par exemple, prédire leur comportement et comprendre pourquoi en général ils agissent ou réagissent comme ils le font. Quant aux choses, cette même psychologie s'en fait une idée qui, vraie ou fausse, permet en tout cas de les fixer et de les déterminer avec suffisamment de fermeté pour que notre comportement envers elles et envers le monde en général soit assuré d'une cohérence et d'une régularité assez fortes que, du reste, confirme pour tout un chacun l'observation du comportement de ses semblables.

Du point de vue qui est le nôtre ici, l'important est que la psychologie du sens commun implique donc aussi ce qu'on pourrait appeler une physique du sens commun au demeurant tout aussi naïve et implicite, mais également tout aussi efficace et assurée que son pendant proprement psychologique. Ainsi, de même que nous avons la conviction de penser et d'être donc capables d'avoir des représentations que nous savons aussi pouvoir partager avec nos semblables, nous vivons parallèlement dans la conviction que beaucoup de ces représentations nous viennent directement d'objets situés dans le monde extérieur et qu'il y a dès lors de bonnes raisons de les tenir pour la cause de nos représentations. Ainsi pensons-nous que si nous avons la sensation du rouge, c'est qu'il y a dans le monde au-dehors de nous des objets de couleur rouge, que si nous entendons des sons aigus, c'est qu'il y a des objets qui émettent un tel son et que, du reste, ceux-ci produisent le même effet sur nos semblables. Il en va de même pour notre identification des choses : si nous voyons des chiens et des chats, le soleil et la lune, c'est que tout cela est comme tel donné de l'extérieur et, pour ainsi dire, tout fait. De toute évidence, la physique du sens commun a un goût certain pour le ready-made, une tendance 
innée à réifier, à peupler l'environnement d'objets auxquels elle reconnait non seulement une existence totalement propre, mais aussi une essence avec toutes les propriétés qui vont avec. Ainsi, un monde d'objets s'impose comme déjà là et sa constitution passe pour indépendante de l'esprit.

Ce qui toutefois ne veut pas dire que la psychologie naïve pense que cette indépendance isole le psychique du physique. C'est au contraire l'objet qui est censé faire la vie de la conscience, comme si la donation du premier avait partout un pouvoir causal sur l'activité de la seconde. Quoi que fasse le sujet, pour la psychologie du sens commun, c'est vers l'objet qu'il est porté car c'est partout l'objet qui appelle le sujet à penser et à se représenter ; c'est la sollicitation du donné objectif qui détermine de part en part la vie psychique. De même que les objets sont supposés se donner à la conscience, de même le sujet est supposé ne consacrer son activité psychique qu'à ce donné et être ainsi avant tout dirigé vers l'objet, ce en quoi justement consisteraient percevoir, se représenter, penser et tout autre acte de conscience. Le dualisme entre le psychique et le physique n'est donc décidément pas la philosophie spontanée du sens commun, car s'il est vrai qu'on est naturellement porté à penser que le monde est constitué d'un ensemble d'objets indépendants de l'esprit, on est $<$ en $>$ même temps porté à penser que l'esprit n'en est jamais pour autant isolé du fait même de sa constante orientation vers ce donné objectif.

Comme le remarque Natorp, la tendance spontanée à comprendre de la sorte les rapports entre l'esprit et le monde réel ne sera certainement pas démentie par la philosophie originaire, lorsqu'elle se donnera au départ pour tâche de clarifier réflexivement les modalités d'exercice de la conscience. Du sens commun, elle héritera en tout cas de la répugnance que l'esprit éprouve naturellement à se considérer comme isolé et coupé du monde. Et elle accentuera d'autant plus cette relation qu'en fixant l'ambition la plus haute de l'esprit dans la vérité ou la connaissance dite objective, elle ne pourra dès lors autrement la concevoir que comme une sorte d'adéquation parfaite entre la représentation de l'esprit et les choses extérieures. Ainsi, répugnant elle aussi au dualisme, la philosophie originaire sera assez naturellement portée sur les voies d'un monisme pour ainsi dire dicté par la fascination profonde de la psychologie naïve pour le monde extérieur, et elle fera très vite une théorie de cette idée que l'exercice de la conscience n'est redevable qu'au fait que des objets lui sont constamment donnés.

Mais, pour Natorp, bien qu'issu d'une entreprise de réflexion, le monisme de la philosophie originaire n'en est pas moins naif que celui spontanément véhiculé par la psychologie du sens commun. Une chose lui échappe en effet : que l'activité de conscience soit tout entière portée vers l'objet devrait au moins laisser à penser que l'objet n'est que dans la mesure où des actes de 
conscience s'y rapportent. Aussi se pourrait-il que la vie psychique soit un présupposé fondamental de l'objet, que l'objet soit constitutivement lié à la conscience et que donc celle-ci ait son mot à dire dans ce qu'on nomme aussi bien la donation ou l'objectivation des objets. Néanmoins, ce sont là autant de voies de réflexion qu'obture le mythe de la réalité forgé par la psychologie du sens commun. Celle-ci sécrète spontanément une physique avec laquelle s'impose l'évidence que les choses nous sont données toutes faites. Or la question de la constitution objective que cette physique naïve a vite fait de régler en l'annulant, la philosophie originaire non seulement ne la relève pas, mais ratifie plutôt ce que fait valoir le sens commun. Le mythe revêt ainsi les allures d'un théorie ontologique. À la psychologie devenue philosophique n'appartient donc pas la question de la fonction constitutive de l'esprit, mais seule lui importe la question de son autoconstitution nécessaire pour accéder à la représentation la plus adéquate possible de la réalité et s'élever de la sorte à la connaissance vraie. Cette question qui, dès le départ, devient la question philosophique par excellence ne peut donc se passer de psychologie, mais, d'obnubiler son propre rapport constitutif à l'objet, cette psychologie incline fortement à traiter l'esprit lui-même comme un objet, celui à propos duquel se pose précisément la question de savoir les façons dont il se rapporte à tous les autres objets qu'il n'est pas. La question de la relation de la pensée aux objets n'est donc posée qu'à l'intérieur d'un horizon ontologique prédéterminé par le sens commun et où la signification du mot être se ramène unilatéralement à celle de l'objet. Telle est la teneur du «monisme naïf» de la philosophie traditionnelle dont Natorp retrouve aisément les traces tout le long du parcours qui mène de la théorie substantialiste de l'esprit chez Aristote à la psychologie naturaliste des modernes.

Certes, le cartésianisme passe communément pour avoir assuré à l'esprit un rôle fondationnel que rendait nécessaire l'approche mathématique de la réalité dans la physique moderne. Nonobstant la mise en valeur de l'autonomie de la conscience qui devait en découler, Descartes ne fournit aucune psychologie qui se porterait jusqu'à la question de savoir ce qu'il en est de la consistance de cette autonomie. Son seul intérêt est la nature, c'est-à-dire tout ce qui de façon générale vaut comme objet, et, partant, sa seule question est de savoir comment, par ses propres ressources, l'esprit peut au mieux s'y conformer. Du reste, il n'est pas à espérer autre chose de la tradition empiriste, même si, ici plus qu'ailleurs, sont clairement reconnus les pouvoirs constituants du sujet sur ce qui n'est au départ qu'un pur donné de sensation. Toutefois, lorsqu'il s'agit d'expliquer ces pouvoirs et de penser ainsi la vie du sujet, on a recours aux mêmes mécanismes que ceux dont on se sert pour expliquer les objets. Ce qui conduit inévitablement à faire du psychique « une seconde

\section{5}

Bull. anal. phén. XV 6 (2019)

https://popups.uliege.be/1782-2041/ @ 2019 ULiège BAP 
nature » à propos de laquelle ne fait que se compliquer la question du rapport qu'elle pourrait avoir avec la nature proprement dite ${ }^{1}$. Dans cette tradition, c'est assurément au kantisme qu'il revient d'avoir ouvert une brèche par un double mouvement de démythologisation de l'objet et de restitution de ses prérogatives au sujet. C'est donc bien de cet héritage que se réclame Natorp, non pas toutefois pour se défaire du monisme mais bien pour le penser autrement que ne le prescrit la psychologie du sens commun.

\section{Du monisme naïf au monisme critique}

La démystification de l'objet exige d'en revenir au fait premier de la conscience sans lequel l'objet ne serait pas et, pour ce faire, $<$ de $>$ commencer par séparer tout ce que confond le monisme naïf. Ce n'est pas démentir l'unité de l'objet et du sujet, mais se donner les moyens méthodologiques de restituer leur constitution réciproque et, par là, de rendre à cette unité tout le sens qu'oblitèrent la psychologie du sens commun et, dans son sillage, la psychologie philosophique traditionnelle. Ce programme d'obédience moniste, Natorp le formule en ces termes :

Le concept d'objectivité lui-même ne peut être définitivement déterminé sans que celui de subjectivité ne le soit. Il est donc nécessaire de reconnaître que le problème de la subjectivité et celui de l'objectivité sont entièrement sur le même plan².

Or c'est sur le chemin du retour au fait même de la conscience que forcément émerge une première controverse avec la phénoménologie de Husserl.

Le fait originaire de $<\mathrm{la}>$ conscience, la Bewusstheit comme la nomme Natorp ou la « consciosité » comme on a choisi de la traduire, dit simplement la relation de la conscience à tout ce dont elle a conscience. Mais, aussi tautologique $<$ que $>$ puisse paraître cette caractérisation fondamentale, elle n'en est pas moins ce que présuppose toute représentation d'objet. Voudrait-on dès lors faire de la conscience à son tour un objet qu'on la laisserait intacte en sa propre présupposition. Si la conscience fait le moi que connaît la psychologie naïve, en revanche celle-ci ignore que le moi originaire ne peut se laisser réduire à un objet. Même en parler comme d'un phénomène ou d'une existence demeure inapproprié puisque chacune de ces façons de traiter la conscience revient à la convertir en un moi dérivé où est déjà perdue son identité première qui est

${ }^{1}$ Ibid., I § 11, p. 39.

${ }^{2}$ Ibid., II $\S 1$, p. 45. 
d'être ce par rapport à quoi il y a quelque chose comme un objet, un donné, un phénomène. "Le moi, dit Natorp, ne peut être objet pour lui-même, car on cesse de le considérer comme moi dès qu'on le conçoit comme objet $»^{1}$. Au fond, si la psychologie se donne pour objet le moi ou la conscience, celui-ci ne saurait être son problème, il est plutôt le «fondement du problème » de la psychologie ${ }^{2}$.

Certes, cette première approche de la conscience n'est encore que négative, puisqu'elle se borne à nous dire que la conscience originaire ne se laisse en aucune façon objectiver sans qu'en soit ipso facto perdue la spécificité. Elle suffit néanmoins à laisser percer une divergence profonde par rapport à Husserl. Pour Natorp, la conscience n'est pas un phénomène et, pour cette raison, elle ne se laisse pas décrire. Une psychologie descriptive au sens de la phénoménologie husserlienne ne peut être concernée que par les contenus de conscience et, du coup, ne saurait atteindre la conscience comme condition de possibilité de ses propres représentations. Tout ce dont elle $<$ est $>$ capable n'est au fond que de se faire une représentation de la conscience, donc de l'objectiver sur la base de ses contenus ou de ses objets de représentation. La phénoménologie se trompe donc de cible et passe à côté de son sujet. Prétendant décrire la conscience, elle ne décrit au juste que ce dont la conscience est la possibilité, à savoir ses représentations ou ses contenus, mais n'atteint nullement par là cette possibilité même, c'est-à-dire la conscience comme telle.

La réplique de Husserl apparaît dès les Recherches logiques, puisque la critique natorpienne de la psychologie descriptive remonte à la mouture de $1888^{3}$, où bien sûr Husserl ne pouvait se trouver visé, ce qui est par contre assez clairement le cas en 1912. Soutenir la thèse de l'indescriptibilité de la conscience, répond Husserl, c'est omettre de considérer que la relation de la conscience aux objets ne s'exerce pas sur un mode uniforme, mais possède au contraire différentes modalités qui font qu'un même objet de conscience ne donne pas forcément lieu à des contenus de conscience identiques. Tantôt ce contenu fait apparaître l'objet comme donné en personne car nous le percevons, tantôt il ne le fait apparaître que comme un objet simplement visé par le truchement d'une signification qui seule alors constitue le contenu de conscience. C'est dire qu'un contenu de conscience n'égale pas toujours l'objet qui est présenté par lui, mais que ce contenu lui-même diffère à raison des différences spécifiques de l'acte qui vise l'objet, c'est-à-dire du mode de

\footnotetext{
${ }^{1}$ Ibid., II $\S 4$, p. 54.

${ }^{2}$ Ibid.

${ }^{3}$ P. Natorp, Einleitung in die Psychologie nach kritischer Methode, Freiburg i. B., $<$ J.C.B. Mohr, > 1888, § 4, p. 13.
} 
relation intentionnelle à cet objet. Parce que le caractère de la relation intentionnelle est, dira-t-on, principalement différent selon qu'il est intuitif ou signitif, il autorise et même exige une description phénoménologique de la conscience en ses différentes essences d'actes. Soutenir l'indescriptibilité de la conscience, comme le fait Natorp, cela revient donc pour Husserl à oblitérer la variété des modalités intentionnelles des actes de conscience qui, en quelque sorte, animent les objets dans la variété des phénomènes ou des contenus de conscience. Bref, si l'on admet le caractère relationnel de la conscience, alors cette relation elle-même doit se laisser décrire à travers la spécificité des différentes modalités de l'exercice intentionnel de la conscience et doivent être mises plus spécialement au jour les différences modales entre son activité de perception et son activité de signification.

Sans même poser ici la question de savoir si la description husserlienne de la conscience selon les deux modalités d'exercice intentionnel que l'on vient de rappeler est tout simplement tenable ou si elle n'est pas plutôt un véritable dogme de la phénoménologie fondé sur une théorie toute faite plutôt que sur une véritable phénoménologie de l'expérience, on concédera à tout le moins que, répliquant comme il le fait, Husserl passe allègrement à côté du sens véritable de l'objection de Natorp. Celui-ci, en effet, ne nie absolument pas que la liaison par laquelle des contenus se présentent à la conscience constitue sa propre marque et qu'au demeurant ces liaisons peuvent être de sortes bien différentes, comme sont donc différentes aussi les façons pour les contenus d'être liés à la conscience. Comme le dit Natorp, « la relation du moi au contenu de conscience n'est nullement indistincte $»^{1}$. Mais, pour Natorp, ce n'est justement pas cela qui nous permet d'atteindre le fond de la conscience, car celui-ci ressortit non pas aux diverses façons d'être en relation au contenu, mais bien à l'unité de cette diversité. Si comme le répète Natorp, la conscience n'est pas le problème de la psychologie, mais le fond du problème, ce fond pose lui-même le problème de sa propre unité : comment la conscience ou le moi demeure-t-il un et identique à travers la diversité de ces contenus ou de ces représentations ? Le seul et unique problème que pose le moi est de savoir comment il peut rester identique à lui-même, dans une unité qui défie le cours de ses représentations. « Le problème, dit Natorp, se trouve dans l'unité ultime, absolument englobante de la conscience $»^{2}$. En fait, avec ce problème, c'est la vraie nature du moi qui se profile, celle de sa dynamique ou de son activité constante. Cette vraie nature ne se laisse comprendre que si on la sort de la statique des faits psychiques et de leur typologie phénoménologique, pour se

${ }^{1}$ Psychologie générale, II $§ 6$, p. 57.

${ }^{2}$ Ibid., II § 7, p. 63. 
confronter à l'énigme de la continuité d'une conscience qui n'œuvre que dans le devenir incessant de ses représentations. Et c'est bien en ce sens que la conscience demeure indescriptible pour Natorp : qui dit description dit donné, mais l'activité ou les activités dont il est ici question ne sont absolument rien de donné1. Il y a donc un paradoxe insurmontable de la psychologie : si c'est bien le subjectif qu'elle recherche au-delà de toute objectivation, elle n'a affaire en ce sens qu'à de l'indéterminé, tant il est vrai que l'objectivation dit partout la même chose que la détermination. Dès lors, demande Natorp : « Comment pourrait-on saisir ce qui est en soi absolument exempt de détermination, sans précisément par là le déterminer - et donc l'objectiver ${ }^{2}$ ? » De la conscience on peut certes dire qu'elle est une activité, mais ceci n'en constitue pas une détermination ; il n'y a pas de transposition, de traduction possible, de l'action de la conscience dans les termes forcément figés d'un quelconque donné et c'est bien pourquoi de la conscience il n'y a au fond rien de plus à dire que son activité. Si l'énigme de son unité persiste néanmoins, sa résolution ne peut être alors être portée qu'au compte de son contenu : c'est à la diversité de mettre en évidence la façon dont elle donne lieu à une unité. Comme l'exprime Natorp :

Le fait d'être conscient ne peut précisément jamais, du point de vue concret, être mis en évidence autrement que relativement au contenu et à la manière dont s'articule celui-ci. L'articulation est précisément celle du contenu, tel qu'il est dans la conscience, à savoir à titre de diversité dans l'unité ; de sorte qu'elle (la conscience) ne peut être précisément mise en évidence que relativement à celle-ci (la diversité dans le contenu) ${ }^{3}$.

L'énigme de l'unité de la conscience n'est donc pas sans solution. Car, bien qu'il n'y ait guère de chance de pouvoir l'éclairer en l'objectivant, c'est pourtant des objets eux-mêmes que la lumière doit venir, exactement de la même façon que seule l'étude minutieuse de la production lithique levalloisomoustérienne peut nous dire quelque chose de la façon de faire de leurs auteurs néanderthaliens. C'est bien là, semble-t-il, une façon d'exprimer encore le monisme de Natorp : on ne peut parler du sujet qu'à travers les objets. Ce qui ne veut certes pas dire que le problème de la subjectivité soit condamné à disparaître dans celui de l'objectivité, mais ce qui veut dire par contre que le problème du sujet ne peut être éclairé qu'en s'orientant vers l'objet. La psychologie n'est donc paradoxalement qu'une théorie de l'objet puisque, en

${ }^{1}$ Ibid., III $\S 4$, p. 69.

${ }^{2}$ Ibid., VIII § 2, p. 221.

${ }^{3}$ Ibid., II $\S 5$, p. 70. 
sa propre activité, le psychique n'est qu'une production incessante d'objets ou une suite d'objectivations et que l'objet, de son côté, est toujours une production déterminée du sujet, c'est-à-dire une subjectivation déterminée. «Dans cette mesure, dit Natorp, objectivation et subjectivation marchent de concert, aucune n'a la priorité sur l'autre $»^{1}$. Mais il reste que l'objectivation est pour ainsi dire la face visible d'une pièce dont la subjectivation est le revers. Aussi l'empreinte du sujet n'est-elle repérable qu'à même l'objet et seule une théorie de l'objet peut servir de moyen à la théorie du sujet. La voie de l'intériorité ou de la subjectivité a pour sens unique l'objet puisque, étant pour ainsi dire cachée, la première est impraticable de façon immédiate, tandis que, de son côté, le second ramène toujours au sujet.

Le dualisme ou l'opposition exclusive du psychique et du physique est donc la plus mauvaise théorie qu'on puisse opposer à la psychologie naïve : il n'y a pas de face à face du sujet et de l'objet, car l'activité de la conscience qui s'inscrit tout entière dans la relation à l'objet interdit de le penser. Mais au monisme du sens commun qui concède tout à l'objet, y compris le sujet, la version de Natorp est d'opposer un principe de réciprocité selon lequel la subjectivité est « la contrepartie de l'objectivité et non pas, en quelque sens que ce soit, une partie de l'objectivité $»^{2}$. Concéder la priorité à l'objet n'est donc ici qu'une exigence de pure méthodologie, en somme dictée par le souci de la meilleure orientation.

Toute présentation d'un objectif est objectivation d'un subjectif, toute présentation d'un subjectif est subjectivation d'un objectif. Il y a entre les deux, pris idéalement, une identité totale - car la seule différence se trouve dans le signe, c'est-à-dire dans le sens de l'examen ${ }^{3}$.

Conformément au bon sens de l'examen qui incline à dire que l'objectivation est toujours première et qu'à partir d'elle la vie du sujet se livre rétrospectivement, tournons-nous donc brièvement vers l'objet et, avant qu'il en devienne un, tournons-nous vers les sensations pour poser la question : comment de celles-ci en vient-on à l'objet?

${ }^{1}$ Ibid., IV § 4, p. 93.

2 Ibid., VII § 1, p. 183.

${ }^{3}$ Ibid., $\mathrm{V} \S 8$, p. 133. 


\section{Le devenir des sensations}

Contrairement à ce que soutiendra Husserl, pour Natorp, le phénomène au sens premier ne pourrait être celui de la conscience pure, ni donc consister en des essences d'actes immédiatement procurées par une vision intellectuelle selon le fameux « principe des principes » défendu au § 24 des Ideen de 1913. Dans la recension qu'il fera plus tard de cet ouvrage, Natorp note à ce propos qu'il se pourrait qu' « une faille apparaisse dès les présupposés les plus fondamentaux et que cette faille soit irréductible $»^{1}$. Nous le savons déjà, pour Natorp, il n'existe aucun donné originaire, fût-il même le corrélat d'une évidence intellectuelle de type platonicien ou d'une intuition d'essence.

Nous n'acceptons... aucun tout-fait... qu'il soit désormais a priori ou empirique $^{2}$.

Si quelque chose peut être appelé donné, il le devient, mais ne l'est pas. Et toute la tâche est de comprendre comment il le devient au cours du processus cognitif de la pensée. "C'est seulement ainsi qu'“il y a", que "se donne"... un donné dans l'élément pur comme dans l'élément empirique $»^{3}$. Au positivisme revendiqué par Husserl comme la marque même de la phénoménologie, il s'agit d'opposer un productivisme dont la radicalité veut aussi que même ce que la phénoménologie revendique comme de l'a priori soit produit ${ }^{4}$. Faut-il entendre par cet a priori quelques fonctions essentielles de la conscience dont la typologie nous serait déjà acquise par une intuition toute spéciale ? Cela n'est qu'un leurre par lequel la phénoménologie ne fait derechef que nous convaincre de son aptitude à faire passer une théorie toute faite pour du phénomène.

Cela dit, n'objectera-t-on pas pourtant que, tout en en amont de l'activité productive de la conscience, des sensations doivent au moins nous être données et, qui plus est, doivent l'être nécessairement pour que la conscience trouve la matière première de son travail d'objectivation? Qu'il y ait donc un datum sensible qui, à l'interstice des premiers échanges entre le psychique et le physique, constitue le degré zéro de la subjectivation, nul ne le contestera. Mais que nous le nommions degré zéro dit bien qu'on ne pourrait le considérer comme un donné au sens où il serait déjà quelque chose de déterminé. Le

$1<\mathrm{P}$. Natorp,> « Les Idées directrices pour une phénoménologie pure de Husserl», trad. franç. de J. Servois, in Philosophie juin 2002, nº 74, p. 19.

${ }^{2}$ Ibid., p. 21.

${ }^{3}$ Ibid., p. 22.

${ }^{4}$ Ibid. 
datum sensible ou le donné hylétique porte sans doute assez mal son nom, car ce n'est qu'en puissance qu'il est une objectivation; sa détermination n'est qu'un possible et « de cette possibilité, la sensation elle-même n'en sait rien, tout comme le nouveau-né, ajoute Natorp, ne sait manifestement rien de ce que la sensation lui donne $»^{1}$. La sensation est certes le point de départ et, à ce titre, « le fondement subjectif de la connaissance objectivante $»^{2}$, mais ce fondement n'est qu'une simple puissance, une possibilité qui ne peut être connue et reconnue comme telle qu'à rebours du procès d'objectivation de la conscience, c'est-à-dire une fois entamée l'actualisation progressive de l'objet. En d'autres mots, c'est une fois atteints le degré de la représentation d'un objet et donc son donné proprement dit que peut apparaitre qu'il n'est qu'une actualisation de ce qui était, en un sens impropre car seulement potentiellement, donné dans la sensation ${ }^{3}$.

Si une généalogie de l'objet et, par sa médiation, du sujet lui-même est possible, alors c'est forcément à la sensation qu'elle ramène. Mais cette généalogie n'est possible que du point de vue où une objectivité a été actualisée et, de ce fait, nous devient aussi évidente que peut l'être un donné. Car qu'est-ce qu'un objet au sens du donné dont on peut avoir une représentation? On répondra certes qu'un objet est le produit de la détermination de ce qui est encore indéterminé dans la sensation et qui donc la rend impropre à véritablement constituer un donné. Mais cette réponse est bien sûr insuffisante tant qu'on ne sait pas en quoi consiste au juste cette détermination ? À cette question, la réponse de Natorp tient en un mot : la mise en relation ou la liaison. C'est de la liaison entre des contenus de sensations, liaison en général suscitée par la proximité spatio-temporelle de ces contenus, que provient la détermination du matériau sensible et, avec elle, une première objectivation donnant lieu à un donné. L'œuvre de la conscience, dit Natorp, consiste en liaison ${ }^{4}$; «c'est la liaison qui possibilise et produit la détermination $»^{5}$. Ce dont la sensation apparaît dès lors comme la potentialité, c'est précisément celle de la liaison, voire éventuellement celle de la séparation, dont l'actualisation correspond à un premier degré de la représentation et, avec elle, à la donation d'un objet. La représentation n'est certes encore ici que celle de l'objet, mais s'agirait-il maintenant de la représentation conceptuelle, peu importe, la conscience a fait montre de sa loi : elle-même ne sera que la détermination de ce

\footnotetext{
${ }^{1}$ Psychologie générale, IV § 11, p.107.

${ }^{2}$ Ibid.

${ }^{3}$ Ibid., IX $\S 5$, p. 254.

${ }^{4}$ Ibid., III $\S 11$, p. 133 .

${ }^{5}$ Ibid., IV $\S 10$, p.105.
} 
qui demeure encore indéterminé dans la représentation perceptive et dont celle-ci offre à son tour la potentialité. Son actualisation dans le concept n'appartient à nouveau qu'à la liaison en unité du divers qu'offre la représentation perceptive.

À travers son exploitation de la notion de relation, Natorp peut très certainement apparaître comme un précurseur des systèmes de constitution d'objets tel qu'on en trouve le prolongement le plus fameux dans l'Aufbau du jeune Carnap en 1928. Mais il est vrai, qu'à l'inverse de ce dernier, sa propre conception de la relation n'est pas encore étayée par les ressources syntaxiques de la logique moderne. La logique des relations de Natorp ressortit davantage à une conception générale des structurations et donc des déterminations objectivantes marquée par la loi tendancielle à l'unité maximale qui, pour Natorp, est la loi même de la raison.

Suite à ce qui vient d'être rapidement esquissé, il ne sera pas très difficile de comprendre le sens de cette loi. Qu'appelle-t-on penser si ce n'est déterminer, et cela de telle façon, selon Natorp, qu'il n'est rien de déterminé pour la conscience qui ne soit son œuvre. Or, cette détermination, la pensée l'exige sans reste, sans restriction, de telle sorte que, par son œuvre, rien ne puisse demeurer indéterminé dans le champ de l'expérience. En celui-ci, le prétendu donné n'est qu'un $\mathrm{X}$ qui est toujours à déterminer quoique, en vérité, il ne soit jamais absolument déterminable, puisque l'objet n'est objet qu'au degré qu'a atteint sa détermination par la pensée. À un degré inférieur, il n'était pas encore l'objet qu'il est et à un degré supérieur il ne le sera plus. C'est dire que si, d'un côté, il ne faut rien admettre comme déjà donné, d'un autre côté, quand le donné advient avec la formation de l'objet, jamais il ne prend l'allure de quelque chose de définitif et d'achevé qui semblerait nous dispenser d'une élaboration plus poussée. Si la connaissance est un processus, si elle est donc toujours en progression et jamais immobile, c'est en quelque sorte qu'elle est mue par le besoin irrépressible de combler le manque qui s'attache à chacune de ses déterminations objectives et qui hante la raison comme son propre contraire, un peu comme ce qui reste d'irrationnel en chacune de ses constitutions ou de ses formations déterminées. Quel qu'en soit le niveau, la conscience d'objet est donc aussi conscience de la relativité de sa connaissance de l'objet et c'est ce qui n'a cesse de motiver sa marche vers une détermination absolue dont l'approche reste forcément asymptotique, comme tendue vers un but qui recule au fur et à mesure que la connaissance progresse et qui, en son absoluité, ne saurait donc être autre chose qu'une pure idée de la pensée ellemême. Le fondement de ce processus est la loi de la raison elle-même ; il s'agit de l'auto-législation conformément à laquelle, poussée par le seul besoin de 
surmonter l'indétermination, la raison n'œuvre qu'à une objectivation toujours plus synthétique et unitaire.

\section{La question des remplissements}

La notion de remplissement n'appartient pas au registre natorpien proprement dit, alors qu'elle est la notion clé de la théorie phénoménologique de la vérité dans les Recherches logiques de Husserl et, à mieux y regarder, sans doute le concept véritablement central de cet ouvrage. Pourtant, la notion intervient à l'occasion chez Natorp qui évoque « un remplissement vivant, concret » de la subjectivité par l'objet ${ }^{1}$. Ce qui n'étonnera pas : il y a du sens à dire que, lorsqu'elle a lieu, l'objectivation ou la détermination d'un donné vient combler l'exigence de la raison, mais à la condition, bien sûr, d'ajouter que ce comblement ne saurait être que partiel et relatif en regard de la loi tendancielle de la raison. Aussi est-ce sous l'égide de cette idée que, lors de sa confrontation avec Husserl au chapitre XI de la Psychologie, Natorp se focalise sur la notion de remplissement pour indiquer qu'étant de nature processuelle, jamais la donation de l'objet ne pourrait avoir le caractère absolu que lui prête Husserl. Natorp le concède, il y a très certainement du juste dans l'idée d'intentionnalité si elle sert à exprimer qu'il n'y a pas d'objet en dehors de l'activité de conscience qui le vise, mais c'est pourtant cette idée que contrecarre celle d'une donation de l'objet dont l'apanage reviendrait à l'intuition ou à la perception.

L'expression du rapport à l'objet comme « intention » est précisément, pour ma conception, tout à fait convenable, car l'objet n'est en effet donné à aucune étape, mais toujours simplement « visé ». Cependant pour cette conception, il n'y a plus de donation figée du «contenu» et donc pas de possibilité d'une description directe de celui-ci - une telle description n'étant que le concept corrélatif d'une telle donation figée. Mais surgit à sa place la « reconstruction » qui n'est que l'envers de la construction de l'objet et qui partage avec elle le caractère génétique ou méthodologique, donc aussi le sens de l'« intention » et certes de l'intention qui ne se remplit jamais².

De son côté, en effet, Husserl soutient, non seulement, la possibilité d'une donation par perception de l'objet, mais aussi l'idée que, lorsqu'elle a lieu, se produit le comblement total de l'intention de signification à vide ou de la représentation par simple signification et que ce moment du comblement ou

${ }^{1}$ Ibid., IV § 4, p. 93.

2 Ibid., XI § 11, p. 318-319. 
du remplissement équivaut à l'accomplissement de la vérité de la représentation en question. Aussi peut-on lire cette suite d'affirmations dans les Recherches logiques:

Toute perception est caractérisée par l'intention d'appréhender son objet comme présent dans son ipséité corporelle. À cette intention correspond éminemment la perception, elle est adéquate quand en elle l'objet lui-même est effectivement, et au sens le plus strict de ce mot, présent en personne (leibhaftig), est appréhendé totalement tel qu'il est, quand par conséquent il est luimême réellement inclus dans l'acte de perception ${ }^{1}$.

Tout d'abord, il y a l'intention de signification, et elle est donnée en ellemême ; c'est ensuite seulement qu'intervient l'intuition correspondante. En même temps, se produit l'unité phénoménologique qui se manifeste, dès lors, en tant que conscience de remplissement. Les termes de « connaissance de l'objet » et de « remplissement de l'intention de signification » expriment ainsi, bien qu'à des points de vue différents, la même situation... Du point de vue phénoménologique, les actes existent dans tous les cas, mais non pas toujours les objets. Le terme de remplissement confère ainsi à l'essence phénoménologique de la relation de connaissance l'expression qui la caractérise le mieux. C'est une donnée phénoménologique première que des actes de signification et d'intuition puissent entrer en cette relation originale. Et quand cela a lieu, quand à l'occasion un acte de l'intention de signification se remplit dans une intuition, nous disons aussi que l'« objet de l'intuition est alors connu au moyen de son concept » ou que « le nom dont il s'agit s'applique à l'objet phénoménal $»^{2}$.

Le remplissement ultime représente un idéal de perfection. Il réside toujours dans une perception correspondante (ce qui suppose bien entendu une extension nécessaire du concept de perception au-delà des limites de la sensibilité). La synthèse de remplissement, dans ce cas, est l'évidence ou la connaissance au sens prégnant du mot. C'est ici que se réalise l'être au sens de la vérité, de la concordance bien comprise, de l'adcequatio rei ac intellectus ${ }^{3}$.

Certes, Husserl n'en est pas dupe, le recoupement d'une perception et d'une signification est un peu moins simple qu'il n'y paraît. Car comment deux choses dont Husserl s'acharne à montrer qu'elles sont si différentes l'une de l'autre pourraient-elles se recouper?

${ }^{1}$ E. Husserl, Recherches logiques, trad. franç. d'H. Élie et alii, Paris, $<$ Puf, $>1972$, tome 2/II, p. 154.

${ }^{2}$ Ibid., tome 3, p. 48-49.

${ }^{3}$ Ibid., p. 15. 
Il ne faut pas seulement, en général, distinguer entre perception et signification de l'énoncé d'une perception, mais reconnaître qu'il ne se trouve même aucune partie de cette signification dans la perception elle-même. La perception qui donne l'objet et l'énoncé qui le pense et l'exprime au moyen du jugement ou plutôt au moyen des «actes de pensée » combinés en l'unité du jugement, doivent totalement être distingués, bien que, dans le cas présent du jugement perceptif, ils aient entre eux la relation la plus intime, qu'ils se trouvent dans le rapport du recouvrement, de l'unité de remplissement ${ }^{1}$.

Pour expliquer le remplissement, il faut donc bien supposer une frange d'homogénéité entre les deux registres, soit que la signification se moule en quelque manière déjà sur le donné de perception, soit, à l'inverse, que le donné de perception s'adapte, en cas de remplissement, à l'intention de signification. Or, selon le scénario de Husserl, la première solution est inenvisageable : l'intention de signification n'est rien d'autre que prétention à la référence et c'est bien pourquoi elle est dite fonctionner à vide. Il ne saurait donc y avoir d'arrangement préalable du sens avec le référent, mais ce n'est qu'une fois mis en présence du référent par une perception que nous pouvons dire qu'il est tel que nous le visions par le sens de notre expression ou de notre jugement. $\mathrm{Ne}$ reste dès lors que la seconde solution : en cas de remplissement, c'est ce que la perception nous livre du référent qui s'adapte à la façon dont le caractérise le sens de nos expressions. Autrement dit, pour que la perception puisse remplir le contenu de signification, il faut nécessairement qu'il y ait en elle quelque chose de proportionné à ce contenu, quelque chose qui soit accordé à la façon dont notre expression ou notre jugement a signifié l'objet. Pour autant qu'elle recoupe la signification, la perception a donc un contenu distinct de l'objet perçu lui-même, et c'est à proprement parler, ce contenu intuitionné qui, en cas de remplissement, recouvre le contenu de signification. Pour exprimer ce contenu d'intuition, Husserl parle de «sens remplissant ». Il s'agit non pas d'un double de l'objet, mais plutôt de ce qui en est perçu et s'avère identique à la signification par laquelle nous le visions.

Nous avons opposé à la signification le sens remplissant (ou aussi à la signification intentionnelle la signification remplissante) en nous référant au fait que, dans le remplissement, l'objet est « donné » intuitivement de la même manière que le vise la simple signification. Nous avons considéré que ce qui, idéalement conçu, coïncide alors avec la signification, était le sens remplissant, et nous avons dit que c'est au moyen de cette coïncidence que la simple intention de

${ }^{1}$ Ibid., p. 36. 
signification, ou encore l'expression, entre en relation avec l'objet intuitif (que l'expression l'exprime, lui et pas un autre) ${ }^{1}$.

C'est in fine par le sens remplissant que Husserl entend démontrer la réalisation de l'adcequatio et étayer ainsi sa propre théorie de la vérité. Vouloir attribuer à cette notion une autre portée dans l'économie de la phénoménologie reviendrait à la détourner de la seule fonction que lui reconnaît Husserl :

Le sens remplissant est conçu comme étant l'essence intentionnelle de l'acte remplissant parfaitement adéquat. Cette formulation est tout à fait correcte et suffit à notre but, qui est... d'exprimer cette évidence capitale que l'essence de signification de l'acte signitif (de l'acte qui exprime) se trouve identique dans l'acte intuitif correspondant, en dépit de la diversité phénoménologique des actes respectifs ${ }^{2}$.

Certes, comme le remarque Natorp, introduire du sens dans le donné de perception aurait pu sembler faire signe vers l'idée qu'il n'y a pas de donation d'objet sans l'activité structurante du sujet ou, en termes plus kantiens, qu'il n'y a pas de perception sans concept. Cette idée aurait dû alors démentir toute prétention à l'accomplissement d'une correspondance au réel, mais c'est justement ce qui ne se produit pas et donc cette même idée qui s'évanouit du fait que tout le dispositif analytique husserlien ne sert au contraire qu'à démontrer cet accomplissement. Natorp l'exprime en ces termes :

Nous reconnaissons toutefois que Husserl s'approche peut-être le plus de l'idéalisme lorsqu'il fait dépendre le contenu perceptif de la pensée, le « remplissement » de l' «intention », la présentation de la représentation et qu'il détermine essentiellement le premier terme par le second - mais avec cette restriction : l'identification est « accomplie », mais elle n'est pas « visée ${ }^{3}$.

Où que l'on cherche les conditions pour qu'une théorie puisse prétendre à l'accomplissement d'une correspondance entre le réel et la pensée, c'est toujours à une certaine conception de la perception que l'on est ramené. Selon cette conception, la pensée peut certes se faire différentes représentations du réel et donner ainsi différentes versions du monde, mais s'il lui est également loisible de distinguer celles qui sont correctes de celles qui ne le sont pas, c'est que l'esprit dispose par ailleurs de cette mesure qu'est la perception ou, en un

${ }^{1}$ Ibid., p. 120. Ce passage de la Recherche VI fait écho à ce qui avait déjà été annoncé aux $\S \S 14$ et 58 de la Recherche I.

${ }^{2}$ Ibid., p. 120.

${ }^{3}$ Psychologie générale, XI § 13, p. 320. 
sens plus large, l'intuition. Bien sûr, pour qu'une telle idée tienne, il ne faut pas que l'intuition puisse elle-même être confondue avec une représentation ; il faut plutôt se la représenter comme une présentation de la réalité ou comme une mise en présence du réel à l'esprit. Pour cela, il n'est que de tenir l'intuition hors du champ sémantique, de l'expurger de tout concept. Le prix de l'opération paraitra d'autant plus abordable qu'elle recueillera sans problème la caution du sens commun. Le gain en revanche est considérable, puisque de décréter l'esprit capable de tabler sur un donné pur, on le suppose également muni d'un critère solide pour évaluer ses propres propositions et ses propres jugements.

Cette thèse est celle de Husserl dans les Recherches logiques, et c'est à sa déconstruction que contribue Natorp à travers sa propre reconstruction du procès de perception. Il y montre, en l'occurrence, que la perception ne recèle rien de particulier qui la rendrait différente du régime de formation conceptuelle propre à la pensée. Elle n'est pas différente de la pensée du fait qu'au lieu de se représenter l'objet elle en serait plutôt la présentation. Derrière cette différence se profile la mythologie des objets donnés et, derrière celle-ci, se trouve dissimulé que la perception est déjà une œuvre de détermination, d'objectivation ou de constitution d'objets. Telle que la définit Natorp, la pensée entame toujours son œuvre par la perception ; c'est ainsi qu'au degré le plus élémentaire de son processus commence la détermination par laquelle elle se donne à elle-même ses premiers objets. Si l'on préfère exprimer les choses à la Goodman ${ }^{1}$, demandons-nous ce que nous percevons quand nous disons voir la lune. La lune est assurément une réalité et il ne fait aucun doute qu'il s'agit là d'une réalité produite par la nature. Mais ce que nous percevons n'est précisément pas "une réalité »; cela reviendrait à se satisfaire de « quelque chose » pour seule réponse à notre question. C'est la lune que nous percevons et il ne s'agit pas là d'une réalité donnée, mais d'un objet que nous constituons par détermination ou, pour le dire plus exactement avec Goodman, il s'agit d'un objet constitué par la version du monde à laquelle nous adhérons. Nous percevons la lune parce que nous l'identifions comme telle plutôt que comme un corps céleste ou comme l'unique satellite naturel de la Terre ou encore comme le cinquième plus grand satellite du système solaire. Ce sont là d'autres déterminations qui font d'autres objets relevant d'autres versions où d'ailleurs ne se trouve plus forcément impliquée la perception. Quant à savoir si tous ces objets ne seraient tout de même pas des représentations différentes

\footnotetext{
${ }^{1}$ Voir à ce propos le petit dialogue improvisé par Nelson Goodman dans « Quelques tracas mondains », trad. franç. de R. Pouivet in $<$ R. Pouivet (éd.), $>$ Lire Goodman. Les voies de la référence, Combas, < L'Éclat,> 1992, p. 17.
} 
d'un même référent, la question paraîtra d'autant plus vaine que ce référent est opaque et d'une opacité que n'atténuent certainement pas les sensations. Encore une fois, il ne s'agit pas par là de nier la réalité, mais à tout le moins de soutenir que ce n'est pas à elle que se mesurent nos représentations. Elles ne sont précédées d'aucune présentation et voudrait-on malgré tout faire endosser aux perceptions cette charge épiphanique que suggère le fait que c'est par elles en général que nous commençons, il resterait que leur présentation de l'objet en est déjà une version et que toutes les perceptions focalisées sur quelque chose de présumé identique ne donnent pas forcément la même version, ni donc le même objet.

La théorie des objets de Natorp offrait déjà de bonnes raisons de penser ce qui ne va pas dans la différence opérée entre signification et perception, elle permet en conséquence de soumettre à révision celle opérée entre signification et référence. Chez Husserl, comme d'ailleurs chez Frege, ce doublet conceptuel est conçu pour servir à résoudre le problème de la vérité. Ce que montre en revanche Natorp, c'est que les conditions de satisfaction d'une représentation ne sont jamais remplies par son adéquation au référent. Pour le dire en termes plus à la mode, il n'y a pas de truthmakers du moins au sens où l'entendent les réalistes qui persistent à faire du référent un ready-made.

Subsiste pourtant la question des conditions de satisfaction de nos représentations, car il est indéniable que toutes n'ont pas pour nous la même validité et que, s'il en est certaines dont nous sommes tout prêts à nous défaire, il en est d'autres, par contre, auxquelles nous continuons apparemment de tenir fermement. C'est bien que, pour une raison ou une autre, les premières nous paraissent douteuses ou franchement fausses, tandis que nous reconnaissons aux secondes une certaine fiabilité. Qu'est-ce qui fait, dans ce cas, la correction ou non de nos représentations?

Si on se réfère à la notion de remplissement, il ne semble pas y avoir de réponses à cette question dans la Psychologie de Natorp, puisque ce n'est certainement pas à en fournir une que sert sa reprise éphémère de la notion. Si la prolifération de nos déterminations objectives n'existe que pour satisfaire l'exigence de la raison, en revanche ces objectivations ne sont jamais satisfaites par un quelconque donné dont on sait désormais qu'il est un mythe. Si ce n'est donc pas de la référence que dépend la vérité de nos représentations, ne serait-ce pas plutôt sur le seul fieri de la raison elle-même qu'il s'agit alors de l'indexer? Ce qui reviendrait à dire que la vérité est une simple exigence logique à laquelle tendent les représentations et, serait-on peut-être tenté d'ajouter, selon le même mouvement qui les mènent de la détermination préscientifique aux conceptualisations propres à la science mathématique de la nature. Mais cette théorie-là ne saurait être la bonne : ou elle ne répond pas 
à la question de la correction des représentations ou elle y répond mal, puisque c'est un fait bien connu que, s'il existe des représentations scientifiques que nous n'avons pas hésité à rejeter à un moment donné, il est par contre des représentations communes auxquelles nous n'imaginons même pas pouvoir nous défaire. Du reste, nous savons aussi qu'à propos d'un même référent nos représentations physicalistes les mieux confirmées ne balaient pas pour autant la validité de certaines représentations doxiques relevant de la plus simple perception. Il s'agit simplement de versions différentes du monde et, en règle générale, une version ne chasse pas l'autre, mais coexistent toutes celles auxquelles nous continuons d'accorder de la valeur, qu'elles soient de type physicaliste ou pas. Quoi qu'en pense Natorp, la validité de nos déterminations objectives n'est donc pas une affaire de degré rationnel de détermination avec son sens + et son sens -, et faudrait-il même admettre une loi tendancielle de la raison à une détermination toujours plus identique et unitaire, nous n'assimilerons pas pour autant cette tendance à un mouvement finalisé vers la connaissance vraie. Aussi y a-t-il sans doute beaucoup à redire aux thèses de Natorp, quand il écrit :

La mathématique offre la possibilité de déterminations toujours plus exactes, d'une façon illimitée, même si elles ne le sont jamais définitivement. Elle offre donc la possibilité de déterminations valides objectivement, même si cette validité n'est jamais absolue, tandis que la perception ne fournit, et ne peut fournir, qu'une présentation subjective - non pas cependant d'un autre contenu, mais bien de ce même contenu objectif. En effet, à l'être susceptible de vérité appartient une unité, une identité de la détermination qui n'est possible, pour les objets des sens, que sur le fondement des mathématiques ${ }^{1}$.

Mais tout ceci ne fait que nous ramener à notre question : si nous pouvons dire, avec Natorp, que la validité de nos représentations n'est pas une affaire d'adéquation au réel, mais aussi, et sans doute cette fois contre lui, qu'elle n'est guère davantage une affaire de gradation conceptuelle de la fonction objectivante, car, tout compte fait, ce n'est pas par comparaison de leur puissance d'unification rationnelle que se jauge la plus ou moins grande validité des représentations, quel peut en être dès lors le critère ? Pour finir, nous voudrions simplement indiquer tout l'intérêt que présente la solution de Husserl, non plus bien sûr celui de la première mais de la dernière œuvre.

${ }^{1}$ Psychologie générale, XI § 19, p. 214. 


\section{La validité des significations}

À en juger par les seuls textes de la Krisis, les vues de Husserl semblent, avec le temps, s'être considérablement rapprochées de celles de Natorp, au point même qu'il est permis de supposer une influence considérable de ce dernier sur tout le développement de la phénoménologie après les Recherches logiques. En témoignent ces lignes, même à défaut de toute référence explicite :

Le sens ultime du reproche que l'on doit faire à la philosophie de tous les temps - à l'exception de la philosophie de l'idéalisme, laquelle cependant manque de méthode — c'est de n'avoir pu surmonter l'objectivisme naturaliste qui fut dès le début et resta toujours une tentation très naturelle. Comme nous venons de le dire seul l'idéalisme sous toutes ses formes a tenté de se saisir de la subjectivité en tant que subjectivité et de faire droit au fait que le monde n'est jamais donné au sujet et aux communautés de sujets autrement que comme valant de façon subjective-relative pour eux, avec ce qui fait chaque fois son contenu d'expérience, et comme un monde qui reçoit toujours dans la subjectivité et à partir d'elle de nouvelles modifications de sens ${ }^{1}$.

Parce que Husserl adhère donc désormais au mot d'ordre néokantien et plus typiquement marbourgeois qui est de ne rien admettre de tout fait ${ }^{2}$, la tâche est devenue, pour lui aussi, de surmonter la psychologie du sens commun et l'objectivisme fondé sur elle pour parvenir à restituer les choses et le monde en général tels qu'ils se constituent dans le processus intentionnel de l'esprit qui les vise. Pour Husserl, tout le sens de la phénoménologie devenue transcendantale est d'exécuter ce programme. Celle-ci représente donc sa contribution originale à ce qu'en écho à une formulation antérieure nous appellerons volontiers la no ready-made theory. Quant à ce que transcendantal veut dire ici, cela peut être facilement éclairé à travers la notion de « subjectif-relatif » maintes fois utilisée dans la Krisis : il ne s'agit plus de considérer les choses naturelles comme simplement objectives, mais, par réduction ou épochè de l'attitude objectiviste, il s'agit de les considérer dans leur relation constitutive à la subjectivité. Or ce que cette relation justement manifeste est que les objets ne le deviennent pour nous que moyennant le contenu de sens de l'acte intentionnel lui-même. Autrement dit, c'est par le sens grâce auquel un acte

${ }^{1}$ La Crise des sciences européennes et la phénoménologie transcendantale, trad. franç. de G. Granel, Paris, <Gallimard,> 1976, p. 300.

${ }^{2}$ Cf. P. Natorp, « Kant et l'école de Marbourg », trad. franç. d'I. Thomas-Fogiel, in $<$ E. Cassirer, H. Cohen, P. Natorp, $>$ L'École de Marbourg, Paris, <Le Cerf, $>1998$, p. 45. 
détermine ou informe les sensations brutes qu'il a de quelque chose que ce quelque chose se constitue comme tel ou tel objet à nos yeux ${ }^{1}$.

Le comble de la difficulté consiste dans le paradoxe des objets intentionnels en tant que tels. Nous entamerons la question en demandant : que sont devenus tous les objets... qui étaient posés avant l'épochè comme réellement existants (ou étant sur le mode du possible, ou aussi bien non-étants), maintenant que dans l'épochè du psychologue la prise de position à l'égard de toute position de ce genre doit être inhibée? Notre réponse est que l'épochè précisément libère le regard non seulement pour les intentions (les « vécus intentionnels ») qui se déroulent dans la vie purement intentionnelle, mais également pour ce que ces intentions posent chaque fois comme valide en elles-mêmes, dans leur propre teneur de sens, en tant que leur objet².

La teneur sémantique ou le sens d'un acte intentionnel est ce que Husserl appelait dans les Ideen un noème. Cependant, la notion de noème dont Husserl faisait pourtant la pièce essentielle de la phénoménologie dans cet ouvrage n'est pas évoquée une seule fois dans la Krisis. Ce n'est pas, bien sûr, que Husserl y ait renoncé, simplement il préfère désormais parler de validité qui n'est qu'une autre façon d'exprimer le sens, mais, semble-t-il, mieux adaptée à ce qu'est devenue sa préoccupation centrale en bout de parcours. Tout à fait indicatif de cela nous semble être cet extrait :

Ce qui est en question de ce point de vue, c'est le monde, non tel qu'il est effectivement, mais tel qu'il vaut chaque fois pour les personnes, le monde qui leur apparaît, avec les propriétés qu'il a pour elles dans cet apparaître : la question est de savoir comment elles se comportent en tant que personnes dans ce qu'elles font et ce qu'elles souffrent... Les personnes sont motivées seulement par ce dont elles ont conscience et grâce aux diverses façons dont, en vertu du sens (Sinn), elles en ont conscience, dont cela vaut ou ne vaut pas pour elles ${ }^{3}$.

De façon plus concise, cet autre extrait dit la même chose :

\footnotetext{
${ }^{1}$ La suite du texte jusqu'à la première citation de la p. 24 inclusivement est reprise dans la conférence de mars 2010 « Textes posthumes III : La contribution de Husserl à la "no ready-made theory" ", Bulletin d'analyse phénoménologique, 15 (2019), 5, p. 17-20. (N. de l'Éd.)

${ }^{2}$ La Crise des sciences européennes, p. 271.

${ }^{3}$ Ibid., p. 327-328. Souligné et légèrement modifié par nous.
} 
Le monde qui est pour nous est celui qui a du sens, et acquiert toujours un nouveau sens pour nous dans notre vie humaine — sens et validité ${ }^{\text {. }}$

Que sens et validité aillent de pair, cela pourrait donc être la clef du problème qui était le nôtre plus haut. Mais comment plus précisément expliquer cette parité ? Pour ce faire, il n'est que de considérer la fonction cruciale qu'occupent nos significations, à commencer par nos significations perceptives, dans l'activité quotidienne. Si cette activité mérite du nom d'expérience, si donc nous ne la menons presque jamais à tâtons, mais au contraire le plus souvent avec cette assurance qui fait que nous $<$ nous $>$ y retrouvons dans les choses et nous débrouillons avec elles en général sans problème, c'est que nous disposons d'un système sémantique qui, quel qu'il soit, est en tout cas suffisamment efficace que pour nous le permettre. C'est de lui que nous tenons de savoir ce que sont les choses, ce que sont leurs propriétés, le genre de relations qu'elles peuvent avoir entre elles, ce que nous pouvons ou pas en attendre. Nous n'avons d'expérience que d'être constamment sauvés de l'opacité du réel grâce à la faculté de mettre immédiatement un nom ou une signification sur la moindre des sensations que nous en recevons. Aussi, hormis les quelques cas très rares où le réel peut nous surprendre, notre expérience ordinaire ne semble nulle part confrontée à l'opacité et, de son point de vue, il serait même incongru de supposer qu'il puisse y avoir quelque chose de tel. Pour elle, au contraire, tout ou à peu près semble transparent : les choses sont ce qu'elles sont, et il ne semble pas opportun de discuter le fait que les chats sont des chats ou que la lune est la lune. Ce qui soutient pourtant toutes ces assurances n'est certainement pas le réel lui-même, mais les significations par lesquelles nous le déterminons dans notre langage ordinaire. Par le sens, non seulement les objets sont constitués, mais ils nous deviennent également obvies, car, d'expérience en expérience, la justesse de nos identifications n'a fait que se confirmer au point que nous sommes pleinement assurés que le monde environnant que nous percevons est bien tel que nous le disons. Cette assurance n'est bien sûr que doxique ; elle repose sur la croyance, l'acceptation ou la présomption que le monde est ainsi fait et qu'il n'y a pas à revenir là-dessus. Ainsi, comme le dit Husserl, il y a une «hypothèse du monde $»^{2}$ et elle n'est soutenue que par notre système sémantique, puisque c'est l'efficacité de ce système, son aptitude à nous orienter au mieux parmi les choses, qui fait la fiabilité de l'hypothèse générale et la confirme jour après jour. Que le sens soit donc ce qu'il y a de plus indispensable à notre expérience

${ }^{1}$ Ibid., p. 294.

${ }^{2}$ Ibid., p. 293. 
s'explique finalement par le fait qu'à travers lui le monde ordinaire, le monde de la vie, nous apparaît comme un sol de validités ou d'évidences constantes. Le sens est valeur car il nous rend le monde valide, plus exactement, il fait en sorte que sa perception ordinaire, comme on dit, tient debout. Certes il y a bien des façons de structurer ainsi le monde et donc de le voir, mais le critère de validité de ces différentes versions ne semble prescrit que par $<\mathrm{le}>$ seul besoin vital de l'expérience, celui de disposer d'une structure efficace pour nous arranger au mieux avec la réalité. Comme en témoignent ces lignes, c'est donc bien sur une note franchement pragmatiste que se clôt la phénoménologie transcendantale ou le constructivisme du dernier Husserl :

L'on ne doit pas avant tout recourir... aux data de sensations prétendument donnés immédiatement, comme si c'étaient eux qui étaient immédiatement caractéristiques des données purement intuitives du monde de la vie. Ce qui est effectivement premier, c'est l'intuition « simplement subjective-relative » de la vie pré-scientifique du monde. Certes pour nous ce «simplement» prend la couleur de la doxa, objet d'un long héritage de mépris. Mais c'est une couleur dont elle ne porte naturellement aucune trace dans la vie pré-scientifique; là elle forme au contraire un royaume de bonne confirmation et ensuite de connaissances prédicatives bien confirmées et de vérités qui sont exactement aussi assurées que l'exige ce qui détermine leur sens : le projet pratique de la vie $^{1}$.

Sur cette base, il resterait dès lors à spécifier la validité des versions physicalistes du monde. Husserl semble indiquer que la science n'est qu'un prolongement de notre vie pré-scientifique et, on le sait, un des leitmotive de la Krisis est précisément de défendre l'idée que, quoi qu'il y paraisse, l'objectivité à laquelle prétendent les prestations de la science continuent de porter la marque du «subjectif-relatif», puisque toutes les productions scientifiques se rattachent ultimement à l'activité concrète de l'homme dans son monde et ce n'est qu'en regard de cette inscription que s'éclaire aussi leur validité. À propos de ces productions, Husserl écrit :

Nous voyons que tous ces résultats théorétiques ont le caractère de « revenus valables » pour le monde de la vie, s'ajoutant en tant que tels au capital de celuici d'une façon permanente... Monde de la vie concret donc, qui serait à la fois le sol fondateur pour le monde «scientifiquement vrai » et à la fois qui l'inclurait dans sa propre concrétion universelle ${ }^{2}$.

${ }^{1}$ Ibid., p. 142.

${ }^{2}$ Ibid., p. 149. 
Est-ce dire que la validité des productions de sens propres à la science n'est elle aussi fonction que de la satisfaction qu'elle peut apporter au besoin pratique de l'expérience humaine et que leur finalité ne se mesure qu'en termes de valeur ajoutée à son efficacité ? Cette conception très moderne et plus spécifiquement baconienne des sciences de la nature n'est certainement pas absente du propos de Husserl. Toutefois, elle s'accorde plutôt mal à cette autre idée, dont visiblement Husserl n'est pas parvenu à se départir, selon laquelle ce serait plutôt l'idéal de vérité qui déterminerait la démarche scientifique en la rendant dès lors purement « théorétique » au sens grec de la theoria, et donc en la détachant complètement de ce que Husserl nomme fort à propos « l'attitude mythico-pratique $»^{1}$. Cette idée ne serait nullement gênante si elle ne concernait que la philosophie ou ce que Husserl appelle encore la science de l'esprit, puisqu'elle n'engagerait en ce cas que la réflexion critique sur les conditions générales de l'expérience humaine dans son monde. L'ennui vient de ce que Husserl impute également à cette démarche purement théorétique et les mathématiques et les sciences mathématiques de la nature sous prétexte qu'il y serait déjà question d'un regard pur sur le monde, délié de tout intérêt pratique car converti au seul souci d'une vérité universellement acceptable. Avec Natorp, et en des termes d'ailleurs assez proches de lui, Husserl continue donc d'entretenir l'illusion d'une « vérité en soi » qui, comme le terme l'indique, n'est en ce qui le concerne qu'un reliquat de l'influence naguère exercée sur lui par la théorie de la science de Bolzano, mais à présent teinté d'une téléologie de la raison manifestement empruntée au kantisme. Mais, en cela, aussi bien la psychologie critique de Natorp que la phénoménologie transcendantale de Husserl ont sans doute encore quelque chose d'inachevé dans leur constructivisme, en d'autres mots, quelque chose qui s'apparente au mythe consistant à penser que les objets construits par la science, et dont elle-même se sert pour peindre la réalité, puissent être autre chose que du «mythicopratique ». Ce serait assurément un autre travail de montrer que, même conçu de façon asymptotique, le thème d'une « vérité en soi » participe lui aussi d'un mythe, qui n'est certes plus celui du donné mais, cette fois, celui de la représentation ou de la signification.

Pour n'en rester qu'à Husserl, s'il y a donc à boire et à manger dans la Krisis, nous n'en retiendrons que l'anticipation de l'idée de la science à laquelle un constructivisme pleinement cohérent nous semble devoir aboutir. C'est une quinzaine d'années seulement après la Krisis que cette idée finira par s'imposer, après que notre modernité philosophique s'est enrichie de notions aussi cruciales que celles de « version » chez Goodman et de " schème

${ }^{1}$ Ibid., p. 365. 
conceptuel » chez Quine. Afin précisément d'illustrer l'idée à laquelle nous faisons allusion, ne citons qu'un passage assez connu de ce dernier :

En ce qui me concerne, en tant que physicien profane, je crois aux objets physiques et non pas aux dieux d'Homère ; et je considère que c'est une erreur scientifique de croire autrement. Mais du point de vue de leur statut épistémologique, les objets physiques et les dieux ne diffèrent que par degré et non par nature. L'une et l'autre sortes d'entités ne trouvent de place dans notre conception qu'en tant que culturellement postulées. Si le mythe des objets physiques est épistémologiquement supérieur à la plupart des autres, c'est qu'il s'est révélé un instrument plus efficace que les autres mythes, comme dispositif d'intégration d'une structure maniable dans le flux de l'expérience ${ }^{1}$.

\section{Note de l'éditeur}

Ce texte, inédit à ce jour, est celui d'une communication de Robert Brisart au colloque Paul Natorp : Questions logiques et psychologiques, organisé le 26 février 2009 à l'Université de Liège. L'organisateur du colloque, Arnaud Dewalque, a un temps projeté d'en publier les actes, avant d'y renoncer pour diverses raisons. Les deux versions du texte que j'ai consultées - le texte original lu au colloque et celui soumis par Brisart en vue de la publication des actes - sont rigoureusement identiques. Mes adjonctions significatives sont signalées par des chevrons simples $(<>)$. (Denis Seron)

${ }^{1}$ W.V.O. Quine, Du point de vue logique, trad. franç. de C. Alsaleh et alii, Paris, $<$ Vrin,> 2003, p. 79. 